Annuaire suisse de politique de développement

\title{
La pratique des partenariats public-privé dans les coopérations allemande et française
}

Xavier Tschumi Canosa

\section{(2) OpenEdition}

Édition électronique

URL : http://journals.openedition.org/aspd/350

DOI : 10.4000/aspd.350

ISSN : 1663-9669

Éditeur

Institut de hautes études internationales et du développement

Édition imprimée

Date de publication : 1 octobre 2005

Pagination : 95-106

ISSN : 1660-5934

Référence électronique

Xavier Tschumi Canosa, «La pratique des partenariats public-privé dans les coopérations allemande et française ", Annuaire suisse de politique de développement [En ligne], 24-2 | 2005, mis en ligne le 18 février 2010, consulté le 08 septembre 2020. URL : http://journals.openedition.org/aspd/350 ; DOI : https://doi.org/10.4000/aspd.350 


\title{
La pratique des partenariats public-privé dans les coopérations allemande et française
}

\author{
Xavier Tschumi Canosa*
}

\section{Contexte}

Les Objectifs du millénaire pour le développement (OMD) sont devenus, pour tous les pays donateurs, le point focal de leurs politiques de coopération internationale. Les «implications politiques, financières et techniques [des OMD] sont considérables $»^{1}$ pour ces pays, dont cependant l'aide publique au développement (APD) tarde à refléter l'engagement politique et stagne manifestement en pourcentage du revenu national brut.

En devant faire toujours plus avec des moyens limités, les bailleurs de fonds ont travaillé à rendre leur aide publique plus efficace en souscrivant, en février 2003, à la Déclaration de Rome sur l'harmonisation, qui vise à simplifier leurs procédures et pratiques en matière de coopération internationale au développement. En mars 2005, les pays du Comité d'aide au développement (CAD) ont entériné la Déclaration de Paris sur l'efficacité de l'aide au développement, basée sur le plan d'action élaboré à Rome et incluant les principes de gestion axée sur les résultats proposés à Marrakech en février 2004.

Cependant, au-delà de la contrainte budgétaire, c'est le rôle même du secteur public qui est remis en question, aussi bien dans les pays donateurs que dans les pays bénéficiaires. Dans cette dynamique, celui-ci est de plus en plus confiné dans des fonctions d'encadrement, alors que le champ opérationnel revient toujours plus au secteur privé, lequel est perçu comme plus efficace et plus compétent dans la réalisation de certains projets de développement que ne pourrait l'être le secteur public.

Tous ces éléments concourent à la mise en œuvre de partenariats public-privé (PPP), qui apparaissent, en effet, comme une réponse naturelle tant aux besoins financiers immenses nécessaires à la réalisation des OMD qu'à la recherche d'une meilleure efficacité de l'aide, tout en réduisant la pression sur les budgets nationaux et le rôle du secteur public en la matière. "Au premier rang des réponses à explorer, figure une logique différente de celle du financement direct des OMD: penser l'APD comme catalyseur des flux de financement du développement, domestiques et internationaux. $»^{2}$

Malgré d'indéniables succès, la pratique des PPP présente toutefois un bilan mitigé et controversé. Quelle importance les PPP ont-ils dans les coopérations

* Collaborateur scientifique à l'IUED.

1 Jean-Michel Severino et Olivier Charnoz, «Les "mutations impromptues": état des lieux de l'aide publique au développement », synthèse de l'article paru dans la revue Afrique contemporaine $\left(\mathrm{n}^{\circ} 213 /\right.$ 2005-1), juin 2005, p. 2.

2 Ibid. 
allemande et française et jusqu'à quel point ceux-ci s'avèrent-ils utiles à la réalisation des OMD, telles sont les deux questions auxquelles tente de répondre cet article.

\section{Le recours aux PPP dans la coopération allemande au développement}

\section{L'organisation institutionnelle de la coopération allemande}

Les partenariats public-privé, en tant qu'instrument de la coopération allemande au développement, s'inscrivent dans un cadre institutionnel et dans une politique qu'il s'agit de décrire ici brièvement avant de les analyser plus en détail dans la section suivante.

Au niveau institutionnel, le ministère de la coopération économique et du développement (Bundesministerium für wirtschaftliche Zusammenarbeit und Entwicklung - BMZ) est responsable de la conception, du suivi et de l'évaluation de la politique allemande de développement.

Pour la mise en œuvre de ses projets de coopération bilatérale, le BMZ confie des mandats à des organismes d'exécution allemands, dont les deux principaux sont la société allemande de coopération technique (Deutsche Gesellschaft für technische Zusammenarbeit - GTZ) et la banque de développement du groupe d'établissements de crédit pour la reconstruction (Kreditanstalt für Wiederaufbau [KfW] Entwicklungsbank).

Dans le groupe $\mathrm{KfW}$, au côté de la banque de développement, il faut encore mentionner la société allemande d'investissement et de développement (Deutsche Investitions- und Entwicklungsgesellschaft - DEG), dont la vocation est de participer (avec des fonds du BMZ) au financement et à la structuration d'entreprises privées dans tous les secteurs de l'économie des pays en développement et en transition.

Au niveau de sa politique, la coopération allemande au développement actuelle repose essentiellement sur un programme d'action intitulé Aktionsprogramm 2015 (AP 2015), approuvé en avril 2001 par le gouvernement fédéral. Ce programme représente la contribution allemande au principal objectif du millénaire pour le développement, qui est de réduire l'extrême pauvreté dans le monde de moitié d'ici à 2015. Il doit encore être rendu opérationnel par une stratégie de mise en œuvre qui, en août 2005, est encore en voie d'élaboration (les pays, les secteurs et les instruments de l'aide allemande y seront alors définis en termes de priorité).

\section{Base et moyens de mise en œuvre des PPP par la coopération allemande}

Au début des années 1990, le BMZ avait conçu un cadre d'orientation pour la promotion du développement économique privé dans ses pays partenaires, s'adressant alors au tissu local d'entreprises. En septembre 1996, il a élaboré un concept transversal de promotion de l'économie privée dans ses pays partenaires ${ }^{3}$. Ce concept remplace le cadre d'orientation et inclut désormais non

3 BMZ, Privatwirtschaftsförderung in den Partnerländern des BMZ. Sektorübergreifendes Konzept, BMZ Aktuell, nº 071, septembre 1996, 14 p. 
seulement les entreprises locales, mais également les allemandes avec lesquelles des formes de partenariat sont déjà présentées.

En 1999, le BMZ a décidé de mettre en œuvre la première phase d'une facilité financière pilote (PPP-Fazilität $)^{4}$, d'une durée de trois ans renouvelable. La deuxième phase de cette facilité a débuté en 2002 et, alors que la première n'était ouverte qu'aux entreprises allemandes, celle-ci est ouverte aux firmes de toute l'Union européenne. Les montants investis par le partenaire privé doivent atteindre au moins $50 \%$ du montant total du projet en PPP et l'engagement des dépenses doit être réalisé dans un délai de trois ans au plus. Les dépenses publiques de la facilité ne sont pour le moment pas intégrées dans les quotas de la coopération bilatérale allemande, technique et financière, bien que cela demeure un objectif à terme.

Selon des lignes directrices établies par le BMZ en janvier $2001^{5}$, la facilité fait intervenir, du côté du partenaire public, la GTZ, la DEG et la KfW et, du côté du partenaire privé, la fondation pour le développement économique et la qualification professionnelle (Stiftung für wirtschaftliche Entwicklung und berufliche Qualifizierung - SEQUA).

L'économie privée allemande dispose également d'un forum de politique de développement (Arbeitsgemeinschaft Entwicklungspolitik der Deutschen Wirtschaft-AGE), qui regroupe des représentants de diverses fédérations (industrie, artisanat, banque, etc.) et dont le but est de coordonner leurs intérêts et de les représenter face aux acteurs politiques et aux organismes publics actifs dans ce domaine de politique. Les journées annuelles organisées par l'AGE, telle celle de $2003^{6}$, sont régulièrement consacrées aux PPP, car ceux-ci sont perçus par le forum comme un modèle de réussite devant devenir un élément central de la coopération allemande au développement. À la fin de la première phase de la facilité en 2002, le BMZ s'est d'ailleurs entendu avec l'AGE pour développer plus avant son programme PPP.

Les projets en PPP mis en œuvre dans le cadre de l'AP 2015 concernent aussi les petites et moyennes entreprises dans les pays partenaires de la coopération bilatérale allemande. Ces petits projets, qui entrent dans le cadre du programme Sud de PPP (Süd-PPP Programm), ont une durée maximale d'un an. Leur part de financement public se situe entre 1000 et 10'000 euros au maximum et leur financement privé correspond à au moins la moitié du montant total. L'administration du projet est effectuée localement.

Les mesures de promotion publique des projets en PPP sont subordonnées à l'examen de cinq critères, dont la pondération est décidée au cas par cas et qui sont aussi les principes de politique de développement du BMZ: respect des droits de l'homme; Etat de droit et sécurité juridique; participation de la

4 «Förderung entwicklungswichtiger Beiträge der deutschen Wirtschaft und ihrer Einrichtungen», in Tilman Altenburg et Tatjana Chahoud, «Bilanz der ersten Jahre», «Schwerpunkt. Entwicklungspartnerschaft mit der Wirtschaft», E+Z, n 4, 2003, p. 144.

5 BMZ, Public-Private-Partnership. Guidelines für Entwicklungspartnerschaften mit der Wirtschaft (PPP), finanziert aus den Haushalttiteln 68711, 68712 oder 68788, Bonn, BMZ, janvier 2001.

6 AGE, Public Private Partnership. Quo Vadis? Chancen und Herausforderungen für die zukünftige Gestaltung von Entwicklungspartnerschaften mit der Wirtschaft, Konferenzbericht, Berlin, novembre 2003, 93 p., disponible sur <www.age-berlin.de>. 
population au processus politique; ordre économique social et ouvert au marché ; orientation de l'action publique vers le développement.

Des conditions supplémentaires ${ }^{7}$ doivent être remplies pour qu'un projet en PPP soit financé par le secteur public:

$\checkmark$ le projet doit répondre aux objectifs de politique de développement définis, tout au moins pour le pays et les secteurs concernés;

๖ les financements public et privé du projet doivent être complémentaires, de manière à ce que, dans le PPP, chaque partenaire puisse atteindre ses objectifs plus efficacement, plus vite et à un moindre prix que s'il entreprenait seul ce projet;

- le financement public du projet ne doit être consenti que si le financement privé en dépend, soit si ce dernier ne serait pas déboursé sans le premier (subsidiarité du financement public);

$\checkmark$ le projet de PPP doit répondre aux exigences de neutralité et de transparence dans le jeu de la concurrence entre entreprises privées;

๖ le financement privé doit être aussi élevé que possible par rapport au financement public (maximisation de l'effet de levier).

\section{Regard critique sur les PPP de la coopération allemande}

La PPP-Fazilität ne finance pas, de loin, tous les projets de développement en PPP. Selon le douzième rapport de politique de développement du gouvernement allemand ${ }^{8}$, plus de 1700 projets de développement en PPP au total ont été entrepris entre 1999 et 2004, avec un volume global de 8,5 milliards d'euros, dont 5,5 milliards ont été mobilisés par les partenaires privés et 3 milliards par les partenaires publics. En ces termes, les PPP ainsi mis en œuvre semblent indéniablement constituer un succès, particulièrement pour ce qui est de l'effet de levier du financement public sur le financement privé.

De ce total des apports publics et privés, environ 700 petits projets ont été financés par la PPP-Fazilität, à hauteur de (seulement) 300 millions d'euros. Environ 800 projets plus importants de coopération bilatérale technique et financière ont été financés en PPP pour un montant de plus d'un milliard et demi d'euros. Le reste des projets, soit 234, sont ceux de la DEG et représentent près de 6,7 milliards d'euros.

Ces chiffres soulèvent un certain nombre de questions, dont les réponses nuancent fortement le succès apparent des PPP mis en œuvre dans le cadre de la coopération allemande au développement. Uwe Hoering, journaliste indépendant spécialiste des questions de politique de développement, en identifie trois principales ${ }^{9}$.

7 BMZ, Gemeinsam Entwicklung gestalten, Partnerschaften zwischen privater Wirtschaft und öffentlicher Entwicklungszusammenarbeit, Bonn, BMZ, 2001, p. 5.

8 BMZ, Zwölfter Bericht zur Entwicklungspolitik der Bundesregierung, Materialien, $\mathrm{n}^{\circ}$ 131, Bonn, BMZ, mai 2005, pp. 170-171.

9 Uwe Hoering, «Panacea PPP: Public Private Partnerships in German Development Co-operation» (synthèse de «Zauberformel PPP». «Entwicklungspartnerschaften» mit der Privatwirtschaft. Ausmass - Risiken - Konsequenzen, WEED Arbeitspapier, Bonn, World Economy, Ecology and Development [WEED], octobre 2003, 46 p.), p. 2. 
La première de ces questions concerne les projets de la DEG, qui représentent près de $80 \%$ de la somme totale financée en PPP, mais qui sont en fait, pour la plupart, des mesures conventionnelles de financement d'investissements des entreprises privées. Ces projets sont-ils véritablement des projets en PPP et, le cas échéant, répondent-ils aux critères et conditions évoqués ci-dessus? Dans une certaine mesure certainement, mais il semble pourtant difficile de garantir qu'ils répondent aux objectifs de politique de développement, condition clé imposée par les lignes directrices fixées par le BMZ en matière de PPP: "In jedem Einzelfall müssen entwicklungspolitische Zielsetzungen Ausgangspunkt für die PPP-Massnahmen sein. ${ }^{10}$ Une étude sur les alliances stratégiques entre la coopération allemande au développement et l'économie privée cite différents exemples impliquant la $\mathrm{DEG}^{11}$. La plupart d'entre eux possèdent, il est vrai, une composante de développement, le plus souvent dans le domaine de la protection de l'environnement et des ressources naturelles, mais leur description donne la très nette impression que le principe de subsidiarité du financement public n'est pas toujours respecté12.

La deuxième question touche à la part du financement privé dans les projets de coopération bilatérale menés par la GTZ et la banque de développement du KfW. La mobilisation de ressources privées pour ces projets financés en PPP a été, dans les faits, très limitée (quelques dizaines de millions d'euros sur un total d'un milliard et demi). Cette situation contraste considérablement avec celle présentée initialement. La mobilisation des ressources privées dépend des secteurs concernés par chaque projet, les infrastructures ainsi que la desserte en eau et son évacuation étant sans doute ceux pour lesquels le financement du secteur privé est le plus fréquent (les statistiques de la banque de développement du KfW indiquent par exemple qu'un projet de coopération financière sur deux dans le secteur de l'eau fonctionne selon une logique de PPP).

La troisième question est liée à l'objectif de réduction de la pauvreté, que la coopération allemande, comme d'ailleurs toutes celles des pays du CAD, a mis en tête de son agenda politique. Force est pourtant de constater que les secteurs de la santé, de l'éducation ou du développement rural par exemple ne sont que très marginalement représentés dans la liste des projets financés en PPP, alors qu'ils se révèlent d'une importance cruciale dans la réalisation de cet objectif. Il en va de même des pays dans lesquels ces projets se déroulent. Ce ne sont pas les pays les plus pauvres, mais des pays qui, a priori, auraient accès à d'autres sources de financement que celles induites par le mécanisme de PPP. "These two indicators (countries, areas) reveal that, by and large, the public

10 «Pour chaque cas particulier, les objectifs de politique de développement doivent être le point de départ des projets en PPP»(notre traduction), in BMZ, Public-Private-Parnership. Guidelines für Entwicklungspartnerschaften mit der Wirtschaft (PPP), op. cit., p. 1.

11 Elke Demtschück, Strategische Allianzen zwischen Wirtschaft und Entwicklungszusammenarbeit, DIE Studies, $n^{\circ} 5$, Bonn, Deutsches Institut für Entwicklungspolitik, 2004, 144 p.

12 Le projet d'utiliser des fibres de coco et du caoutchouc naturel dans la production de pièces automobiles au Brésil est un bon exemple. Des petits paysans ont certes pu participer à la culture écologique de ces intrants naturels et un certain nombre de places de travail a effectivement été créé dans la fabrique, équipée et financée par la filiale brésilienne de Mercedes-Benz. Si, comme c'est le cas, $87 \%$ du coût du projet sont supportés par le partenaire privé, est-ce que ce dernier n'aurait pas entrepris ce projet, même sans le financement public qui l'accompagne? 
funds follow the private flow of investments instead of helping to channel these investments to where they would be required for poverty alleviation. $»^{13}$

L'exemple du partenariat stratégique entre la GTZ et le cimentier suisse Hol$\mathrm{cim}^{14}$ est typique de ces PPP dont l'objectif ne concourt que de loin à l'objectif principal du millénaire pour le développement. Signé en 2003 pour trois ans et doté d'un cadre financier de 960'000 euros supporté à part égale par les deux partenaires, ce partenariat vise à l'utilisation comme combustible des déchets hautement énergétiques issus du processus de fabrication du ciment et contribue ainsi à remédier aux problèmes écologiques et sanitaires engendrés auparavant par ces déchets. Il se déroule dans quatre pays à revenu intermédiaire (selon la classification du CAD): le Chili, le Mexique, le Maroc et les Philippines. Bien qu'il ne faille pas nier les effets positifs que la réalisation des projets de ce partenariat stratégique induit et continuera d'induire sur différents plans (notamment environnemental), il apparaît clairement qu'en termes de réduction de la pauvreté, les effets seront sans doute insignifiants.

\section{Le recours aux PPP dans la coopération française au développement}

\section{Le dispositif français de coopération internationale au développement}

Pour la France aussi, il est utile de présenter d'abord brièvement l'organisation institutionnelle et les stratégies de sa coopération au développement, avant d'examiner plus minutieusement la place donnée aux PPP dans ce cadre.

Les deux pôles du dispositif de coopération française sont le Ministère des affaires étrangères (MAE) et le Ministère de l'économie, des finances et de l'industrie (MINEFI). Pour assurer la coordination entre ces deux pôles, le Comité interministériel de la coopération internationale et du développement (CICID) détermine les orientations stratégiques de la politique d'aide de la France de même qu'il suit et évalue la conformité de celle-ci aux objectifs définis.

Une autre tâche de ce comité est de délimiter la zone de solidarité prioritaire $(\mathrm{ZSP})^{15}$, dans laquelle l'Agence française de développement (AFD) joue son rôle d'opérateur pivot des projets bilatéraux de coopération. Placée sous la tutelle conjointe des deux ministères clés du dispositif, l'AFD a la vocation de banque de développement en faveur des pays de la ZSP et la responsabilité de la mise en œuvre des projets d'aide dans certains domaines, notamment l'appui au secteur privé.

Dernier élément du dispositif français de coopération, le Haut Conseil de la coopération internationale (HCCI) regroupe quelques élus nationaux et locaux mais surtout des représentants de la société civile engagés dans l'effort français

13 «Ces deux indicateurs (pays, secteurs) révèlent que dans l'ensemble les fonds publics suivent les flux d'investissements privés au lieu d'aider à orienter ces investissements là où ils seraient nécessaires à la réduction de la pauvreté» (notre traduction), in Uwe Hoering, op. cit., p. 2.

14 Voir, dans le présent dossier, «L'importance des partenariats multisectoriels pour le secteur privé. l'expérience de Holcim », de Stefanie Koch.

15 La ZSP rassemble actuellement 54 pays parmi les moins développés, principalement en Afrique. La liste de ceux-ci est disponible sur la page Internet suivante du MAE: <www.diplomatie.gouv.fr/ solidarite/fsp/zone.html>. 
de solidarité internationale au sens large. Le but du HCCI est de favoriser la concertation entre les différents acteurs de la coopération internationale et de sensibiliser l'opinion publique française aux enjeux de cette coopération.

Au niveau de sa politique de développement, la contribution de la France à la réalisation des OMD se traduit concrètement par l'élaboration de stratégies sectorielles pluriannuelles ${ }^{16}$ dans sept secteurs d'intervention prioritaires: l'éducation, l'eau et l'assainissement, la santé et la lutte contre le sida, l'agriculture et la sécurité alimentaire, les infrastructures en Afrique subsaharienne, la protection de l'environnement et de la biodiversité et, finalement, le développement du secteur productif. Lors de la sixième réunion du CICID, en mai 2005, il a été décidé que le MAE élabore encore une huitième stratégie dans le secteur de la gouvernance mais celle-ci n'est, en août 2005, pas encore disponible.

\section{Les PPP comme axe stratégique}

\section{de la coopération française au développement}

«La politique française de coopération s'inscrit dans une très longue histoire qui ne peut être séparée de son passé colonial, des étroites relations entretenues entre les pouvoirs publics et les intérêts privés, mais, plus généralement, elle se rattache à la place que la France entend prendre sur la scène internationale. ${ }^{17}$

Les sept stratégies sectorielles de la politique française de coopération au développement font toutes référence à une forme ou à une autre de partenariat public-privé (PPP). L'AFD considère ce mécanisme comme un axe prioritaire de sa stratégie et relève que «près de la moitié des opérations financées par le groupe (représentant en moyenne 1,5 milliard d'euros par an) auprès de bénéficiaires publics ou privés dans les pays en voie de développement, ainsi que dans l'outre-mer français, concerne des formes de PPP. [...] Le PPP, pour les avantages qu'il présente, est une forme d'intervention que l'AFD entend continuer de promouvoir, dans un souci d'adaptation accrue au contexte et aux objectifs de développement $\gg^{18}$.

Le renforcement de l'AFD en matière de PPP est pensé en termes non seulement quantitatifs, mais également qualitatifs. Ainsi, de nouvelles formes de partenariats élargis avec le secteur privé local et la société civile des pays bénéficiaires de l'aide sont promues, des partenariats dans de nouveaux secteurs comme la formation professionnelle ou l'environnement sont recherchés et de nouveaux instruments financiers - les garanties d'emprunts en monnaie locale par exemple - sont encouragés.

Le recours aux PPP dans les secteurs de l'eau et de l'assainissement est peutêtre le plus important, compte tenu de la pratique centenaire de ce type de partenariat en France même. L'importance, et les limites, des PPP noués dans ces secteurs seront illustrées plus en détail ci-dessous.

16 Ainsi que par une programmation par pays plus sélective et adaptée en fonction de la capacité de ces derniers à gérer l'aide qu'ils reçoivent (logique d'aide fondée sur la performance).

17 Jean-Jacques Gabas (dir.), L'aide publique française au développement, Etudes de la Documentation française, Paris, La Documentation française, 2005, p. 21.

$18 \mathrm{AFD}$, Le partenariat public-privé à l'Agence française de développement. Une approche renouvelée, Paris, AFD, août 2002, p. 8. 
Enfin, les entreprises privées sont aussi de plus en plus nombreuses à intervenir dans le domaine de l'aide humanitaire, même si cette dernière n'est pas guidée par les stratégies sectorielles évoquées ci-dessus. Ces interventions se déroulent le plus souvent en partenariat ou en liaison avec une ONG active et expérimentée dans ce champ. "Les pouvoirs publics encouragent à la fois l'intervention des entreprises dans le domaine de l'humanitaire d'urgence et la conclusion de partenariats, soit avec des ONG, soit avec des collectivités locales. ${ }^{19}$

\section{La position française sur la question de l'eau et de l'assainissement}

Un des OMD, complété lors du Sommet de la Terre à Johannesburg, est spécifiquement dédié à l'eau. Il vise entre autres à «réduire de moitié, d'ici à 2015, le pourcentage de la population qui n'a pas accès de façon durable à un approvisionnement en eau de boisson salubre et à des services d'assainissement de base $»^{20}$.

Pour réaliser cet objectif, le rapport Camdessus ${ }^{21}$, présenté au $3^{\text {e }}$ Forum mondial de l'eau à Kyoto en mars 2003, préconise notamment aux donneurs d'aide de doubler leur financement dans le secteur de l'eau et d'y accroître l'efficacité de l'aide en recherchant, par des multipartenariats, à maximiser l'effet de levier de l'APD sur les autres sources de financement du développement. La France a fait siennes ces recommandations du rapport en juin 2003, lors du Sommet du G8 à Evian, en faisant adopter un plan d'action du G8 pour l'eau et un autre pour l'Afrique, et en annonçant le doublement du financement français pour le secteur de l'eau.

L'année précédente, les acteurs ${ }^{22}$ français de l'eau avaient conçu une charte d'engagement pour l'accès à l'eau et à l'assainissement, qui clarifie le rôle de chacun d'eux, autorités publiques et partenaires (société civile, opérateurs et bailleurs). Les éléments fondamentaux sur lesquels se base cette charte sont, entre autres, que la ressource en eau «ne peut faire l'objet d'une appropriation privée», que «la collectivité publique doit impérativement conserver la maitrise des infrastructures [...] et des principaux réseaux collectifs» et que «le montant des investissements et la technicité des savoir-faire nécessaires supposent [...], si nécessaire, le recours à des partenariats multi-acteurs, entre les pouvoirs publics, les entreprises, privées ou publiques, et la société civile ${ }^{23}$.

Sur cette base, la charte définit le rôle des autorités publiques et la contribution des partenaires dans le contexte local des services d'eau et d'assainissement: l'Etat, autorité régalienne reconnue par les usagers, définit «le cadre juridique, l'organisation des pouvoirs, la mobilisation des ressources financières, la mise

19 Site Internet d'Urgence humanitaire France: <www.diplomatie.gouv.fr/humanitaire/1035.html> $>$ acteurs français >entreprises.

20 CICID, «Stratégie sectorielle eau et assainissement», Paris, mai 2005, p. 1.

${ }_{21}$ Panel mondial sur le financement des infrastructures de l'eau, Financer l'eau pour tous, rapport du panel présidé par Michel Camdessus, 2003, 72 p.

22 Etat et collectivités territoriales, entreprises publiques et privées, agences de l'eau, institutions universitaires et de recherche, ONG, experts, etc.

23 «Charte d'engagement pour l'accès à l'eau et à l'assainissement», in Comité français pour le Sommet mondial du développement durable Johannesbourg 2002, Livre blanc des acteurs français du développement durable, Paris, Premier Ministre, 2002, pp. 41-45, citations p. 42. 
en place de programmes nationaux et de mécanismes de solidarité... pour favoriser concrètement l'accès de tous à l'eau et l'assainissement ${ }^{24}$. A cette fin, il peut compter sur des autorités organisatrices proches du terrain qui veillent à ce que les programmes, les tarifs, les modes de gestion correspondent aux réalités et attentes locales. Au niveau de la contribution de chaque partenaire, la charte précise celle des usagers, qui doivent tout à la fois communiquer leurs demandes, évaluer et contrôler le service qui leur est fourni. Elle exige ensuite que les opérateurs privés honorent les termes du contrat dans le long terme (pérennité du service et protection de l'environnement). Enfin, la contribution demandée aux bailleurs de fonds par la charte est de conforter la maîtrise d'ouvrage par l'autorité locale et de lui donner les moyens de fournir ses services et de tenir ses engagements contractuels de délégation.

En mars 2004, sous l'égide du Comité des affaires internationales de l'Association scientifique et technique pour l'eau et l'environnement (ASTEE), les acteurs français de l'eau ont rédigé un document ${ }^{25}$ qui synthétise leurs initiatives communes pour réaliser les OMD: inciter les gouvernements bénéficiaires de l'aide à définir leurs politiques nationales de l'eau, organiser une gestion concertée de la ressource rare en eau (pour éviter les conflits liés à cette ressource), promouvoir une charte internationale d'accès aux services d'eau et d'assainissement et de bonne gouvernance (texte en préparation au niveau européen), impliquer les collectivités locales, apporter les financements et garantir l'investissement (en diversifiant notamment les instruments de financement susceptibles d'avoir un effet de levier sur les ressources mobilisables), accroître la solidarité entre les utilisateurs de l'eau et mesurer les progrès au travers d'un «observatoire de l'eau » (l'Organisation internationale de normalisation [ISO] travaille sur un système d'indicateurs).

Pour l'AFD, la desserte en eau constitue un défi stratégique. Elle considère que, pour le secteur de l'eau et de l'assainissement, des PPP de longue durée doivent s'instaurer dans «un environnement institutionnel adapté, comportant:

$\checkmark$ une composante de régulation sectorielle;

$\checkmark$ une forte implication des partenaires et une relation aussi équilibrée que possible, dans un cadre contractuel non figé;

$\checkmark$ un partage des risques adéquat: le risque doit être affecté à celui des deux partenaires qui est le mieux à même d'y faire face ;

$\checkmark$ un partage des coûts permettant de réaliser des projets à rentabilité financière faible, mais assortis d'effets externes positifs suffisamment importants $»^{26}$.

24 Ibid., p. 43.

25 Comité des affaires internationales de l'ASTEE, Accès à l'eau et à l'assainissement. Mobilisation des acteurs français pour un défi mondial!, Paris, ASTEE, mars 2004, 8 p.

$26 \mathrm{AFD}$, «La desserte en eau, un défi stratégique pour l'Agence française de développement», août 2002, p. 3, tiré du site Internet de l'AFD, <www.afd.fr> >publications > autres publications. 
En matière de PPP dans le domaine de l'eau, la logique contractuelle la plus répandue est la gestion déléguée de l'eau, qui confie aux opérateurs privés tout ou partie de l'exploitation des infrastructures mais permet à la collectivité locale de conserver ses pouvoirs d'autorité, d'organisation et de contrôle (notamment des tarifs) sur le service. Ce système est typique de l'expérience française et remonte aux premiers réseaux urbains d'eau dans ce pays (les trois quarts de la distribution d'eau en France reposent actuellement sur ce mode de gestion). C'est d'ailleurs de cette longue expérience que les multinationales françaises ont réussi à forger leur domination sur le marché mondial de l'eau.

En tant qu'outil de coopération internationale, la gestion déléguée s'est progressivement imposée aux dépens notamment de la «privatisation pure et dure ${ }^{27}$ des infrastructures de l'eau, longtemps préconisée par la Banque mondiale mais finalement délaissée au profit de modes de gestion mixte «à la française ${ }^{28}$.

Cependant, deux difficultés majeures se posent lors de la diffusion d'un tel mode de gestion dans les pays en développement. La première concerne «le déséquilibre de compétence entre l'autorité locale délégante et le délégataire privé ${ }^{29}$. En effet, l'autorité locale dispose d'une marge de manœuvre réduite par rapport à l'opérateur privé puisqu'elle doit d'une part lui relayer les attentes des populations et, d'autre part, leur rendre des comptes quant à l'offre fournie.

La deuxième grande difficulté est «l'absence de régulation d'ensemble du marché des services d'eau à l'échelle du pays ${ }^{30}$. Avant que cette difficulté ne soit levée, le débat sur l'intervention d'opérateurs privés dans la fourniture de services essentiels comme l'eau et l'assainissement apparaît comme un faux débat car ces opérateurs n'ont ni la capacité, ni l'obligation, ni même la vocation d'assurer ces services de manière universelle en faveur des populations des pays en développement. "A ce titre, invoquer des gains d'efficacité liés à la privatisation des services de l'eau est peu crédible, sauf à poser comme préalable la mise en œuvre efficace d'une législation adaptée et d'une régulation opérante ${ }^{31}$.

Ainsi, dénoncer dans le cadre du Forum alternatif mondial de l'eau (FAME) «le discours unique de l'oligarchie de l'eau qui prône le recours au privé comme la seule solution désormais envisageable $»^{32}$ ou «les risques croissants de pareille "marchandisation" d'un bien commun de l'humanité »"33 est certes nécessaire mais non suffisant. Indéniablement, les grands opérateurs privés dans les secteurs de l'eau et de l'assainissement ont des appétits d'investisseurs dans cer-

27 Entretien avec Jean-Noël Roulleau, «L'ambition française», Courrier de la Planète. Eau - Inventer la coopération internationale, $\mathrm{n}^{\circ} 70,2002$, p. 53.

28 Ibid.

29 Damien Conaré, Elie Cohen et Henri Smets, «Vers une autorité mondiale de l'eau?», in Thierry Giordano (coord.) et al., Eau: encadrer les partenariats public-privé, Les Notes de l'IDDRI, Paris, Institut du développement durable et des relations internationales (IDDRI), 2003, p. 32.

30 Ibid.

31 Thierry Giordano, «Avant-propos», in Scott Vaughan, Privatization, Trade Policy and the Question of Water, Les Séminaires de l'IDDRI, nº 9, Paris, IDDRI, 2003, p. 7.

32 Marc Laimé, «La "titrisation" du patrimoine public. Une nouvelle piste de financement de l'accès à l'eau et à l'assainissement dans le monde», contribution au FAME 2005 sur «L'eau bien public, finance publique», p. 2 .

33 Ibid. 
tains pays en développement bien précis (la Chine par exemple), laissant de côté tous les autres (notamment les pays les moins avancés [PMA]), et le risque existe qu'ils ne se concentrent que sur les secteurs marchands lucratifs, sans répondre aux demandes bien réelles mais insolvables des populations.

Aussi, pour éviter au maximum ces scénarios, une autorité mondiale de l'eau est-elle appelée à être créée, notamment lors du $3^{\mathrm{e}}$ Forum mondial de l'eau à Kyoto en 2003. Cette autorité répondrait aux deux difficultés évoquées ci-dessus, en vérifiant la transparence des procédures d'attribution des contrats puis de leurs termes, et en fournissant aux opérateurs privés la sécurisation nécessaire à leurs investissements dans les pays en développement qui ne pourraient en bénéficier sinon. Une autre tâche de cette autorité serait de mesurer l'avancement des efforts internationaux par rapport aux OMD, particulièrement celui sur l'eau et l'assainissement. La France favorise la création d'une telle autorité dans ce sens, mais les questions liées à la souveraineté nationale, notamment, rendent les négociations difficiles ${ }^{34}$.

\section{Conclusion}

L'examen de la pratique des PPP dans les coopérations allemande et française à la lumière des OMD permet de tirer trois conclusions principales.

La première tient à l'importance grandissante prise par cet instrument depuis la définition des OMD. La Conférence de Monterrey sur le financement du développement, qui s'est tenue en mars 2002, préconise l'engagement de ressources privées dans l'effort international de réduction de la pauvreté. Les OMD ont été quantifiés en termes de besoins financiers et le PPP apparaît alors comme un moyen privilégié de mobiliser les investissements nécessaires à cette fin. L'Accord général sur le commerce des services, en négociation dans le cadre de l'Organisation mondiale du commerce (OMC) et dont l'objectif est la libéralisation progressive du commerce des services, constitue un élément d'influence additionnel quant à l'importance, accrue ou pas, que prendra le PPP dans la coopération internationale au développement. Pour le moment, cet outil a indéniablement le vent en poupe.

La deuxième conclusion touche à la raison principale qui modère l'enthousiasme suscité par l'instrument PPP. En amont de l'intervention du secteur privé dans les opérations en PPP, le cadre institutionnel est souvent déficient dans les pays récipiendaires et là se situe incontestablement le point de départ de l'échec de ces opérations dans une perspective de réduction de la pauvreté telle que visée par les OMD. Ces déficiences ouvrent le champ à toutes sortes d'abus, de la part des opérateurs privés comme des autorités locales d'ailleurs. Comment remédier à cette situation est sans aucun doute la première question à laquelle la communauté des pays donateurs doit répondre. Des efforts sont en cours.

La troisième conclusion est plus fondamentale puisqu'elle relève de la cohérence des pays donateurs dans la conduite de leurs politiques d'aide au développement. Celles-ci ont été façonnées pour répondre aux OMD, dont la réalisation

34 Voir, dans le présent dossier, «Le partenariat public-privé dans le secteur de l'eau au Niger: autopsie d'une réforme», de Mahaman Tidjani Alou. 
constitue encore à ce jour un engagement international. Atteindre ces objectifs ambitieux devrait alors requérir, de la part des pays donateurs, toute leur attention et l'engagement de tous les moyens à leur disposition, y compris les projets en PPP. Lorsque de tels projets sont mis en œuvre, le partenaire public, pour garantir la cohérence de sa politique de développement, doit veiller à ce que le partenaire privé partage la primauté de l'objectif de développement qui est à la base du PPP. Certes, des intérêts privés sont en jeu, mais ils ne devraient pas aller à l'encontre des objectifs de coopération internationale, principalement les OMD. Pour assurer cela, un mécanisme de régulation apparaît comme un élément crucial du partenariat et comme le garant d'une meilleure cohérence et d'une plus grande efficacité de l'aide.

\section{Bibliographie}

\section{Allemagne}

AshofF, Guido, «Germany's Development Co-operation Policy since the Early 1990s: Increased Conceptual Ambitions in Times of Severe Financial Constraint», in HoEbink, Paul, Stokke, Olav (dir.), Perspectives on European Development Co-operation: Policy and Performance of Individual Donor Countries and the EU, London; New York, Routledge, 2005, pp. 267-302.

BundESMINISTERIUM FÜR WIRTSCHAFTLICHE ZUSAMMENARBEIT UND ENTWICKLUNG (BMZ), Zwölfter Bericht zur Entwicklungspolitik der Bundesregierung, Materialien. nº 131, Bonn, mai 2005, 299 p.

COMITÉ D'AIDE AU DÉVELOPPEMENT (CAD), Examen en matière de coopération pour le développement. Allemagne 2001, Les Dossiers du CAD, vol. 2, nº 4, Paris, OCDE, 2002, 68 p.

DeмtSснÜск, Elke, Strategische Allianzen zwischen Wirtschaft und Entwicklungszusammenarbeit, DIE Studies, $n^{\circ}$ 5, Bonn, Deutsches Institut für Entwicklungspolitik (DIE), 2004, 144 p.

Hoering, Uwe, «Panacea PPP: Public Private Partnerships in German Development Co-operation» (synthèse de «Zauberformel PPP». «Entwicklungspartnerschaften» mit der Privatwirtschaft. Ausmass - Risiken - Konsequenzen, WEED Arbeitspapier, Bonn, World Economy, Ecology and Development [WEED], octobre 2003, 46 p.), 6 p.

\section{France}

AGENCE FRANÇAISE DE DÉVELOPPEMENT (AFD), dossier «Les "mutations impromptues": état des lieux de l'aide publique au développement», Afrique contemporaine, $\mathrm{n}^{\circ}$ 213, 2005, pp. 13-131.

Comité d’aide au développement (CAD), Examen par les pairs. La France, Paris, OCDE, 2004, $115 \mathrm{p}$.

CHASSIRON, Anna (DE), «La politique française d'aide au développement: la réforme de 1998 face aux défis de demain», mémoire, Paris, Ecole nationale d'administration, février 2004, 57 p.

Gabas, Jean-Jacques, «French Development Co-operation Policy», in HoEbink, Paul, STокKe, Olav (dir.), Perspectives on European Development Co-operation: Policy and Performance of Individual Donor Countries and the EU, London; New York, Routledge, 2005, pp. 242-266.

- (dir.), L'aide publique française au développement, Etudes de la Documentation française, Paris, La Documentation française, 2005, $171 \mathrm{p}$. 